\title{
Article \\ Prescribed Performance Control for the Upper-Limb Exoskeleton System in Passive Rehabilitation Training Tasks
}

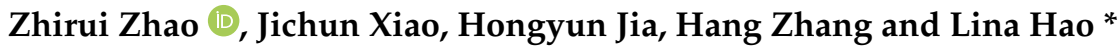 \\ Department of Mechanical Engineering and Automation, Northeastern University, \\ Shenyang 110819, China; 1710091@stu.neu.edu.cn (Z.Z.); 2010082@stu.neu.edu.cn (J.X.); \\ 2110084@stu.neu.edu.cn (H.J.); 17824220563@163.com (H.Z.) \\ * Correspondence: haolina@me.neu.edu.cn
}

Citation: Zhao, Z.; Xiao, J.; Liu, H.; Zhang, H.; Hao, L. Prescribed Performance Control for the Upper-Limb Exoskeleton System in Passive Rehabilitation Training Tasks. Appl. Sci. 2021, 11, 10174. https:// doi.org/10.3390/app112110174

Academic Editor: Claudio Belvedere

Received: 30 September 2021

Accepted: 27 October 2021

Published: 29 October 202

Publisher's Note: MDPI stays neutral with regard to jurisdictional claims in published maps and institutional affiliations.

Copyright: (c) 2021 by the authors. Licensee MDPI, Basel, Switzerland. This article is an open access article distributed under the terms and conditions of the Creative Commons Attribution (CC BY) license (https:// creativecommons.org/licenses/by/ $4.0 /)$.
Abstract: In this study, a model-free adaptive sliding mode control method was developed in combination with the prescribed performance method. On this basis, this study attempted to fulfill the joint position tracking trajectory task for the one-degree of freedom (DOF) upper-limb exoskeleton in passive robot-assisted rehabilitation. The proposed method is capable of addressing the defect of the initial error in the controller design and the application by adopting a tuning function, as compared with other prescribed performance methods. Moreover, the method developed here was not determined by the dynamic model parameters, which merely exploit the input and output data. Theoretically, the stability exhibited by the proposed controller and the tracking performance can be demonstrated. From the experimental results, the root mean square of the tracking error is equal to 1.06 degrees, and the steady-state tracking error converges to 1.91 degrees. These results can verify the expected performance of the developed control method.

Keywords: upper-limb exoskeleton; model-free adaptive control; prescribed performance; passive rehabilitation

\section{Introduction}

The exoskeleton refers to a wearable robot system comprising an external structural mechanism with joints and links corresponding to the human body [1]. With the initial advent of the exoskeleton robot, termed "Hardiman" and designed by the General Electric company born in 1960, people have quickly undertaken the development of wearable robot systems to assist and augment the force of human physical motions [2]. The Berkeley lower extremity exoskeleton (Bleex), the hybrid assistive limb, and the "Mindwalker exoskeleton" were designed for soldiers, nurses, and the elderly, respectively, to enhance their movement abilities and improve their loading limitation [3-6]. In addition, these have been extensively used for robot-assisted rehabilitation therapy for the elderly or victims of stroke, spinal cord injury, and athletic injuries (e.g., SUEFUL-7 and EXO-7) [7-9]. As strongly demonstrated by related works, robot-rehabilitation exercises are capable of facilitating the recovery of the physical activity functions of patients, which can be longer lasting and repeatable [10-13]. Moreover, upper-limb exoskeletons are primarily driven by servo motors (e.g., high radio reducers) or hydraulic systems with a bulky size, poor portability, as well as high joint stiffness. The actuator of the upper-limb exoskeleton should be a high power-to-weight ratio and exhibit a soft but flexible structure [14-17]. To this end, a pneumatic artificial muscle (PAM) has been proposed for exoskeletons which is consistent with human muscles as it exhibits a flexible structure and is a rich carrier of energy [18-21]. PAM is operated by air pressure. Its core element refers to a reinforced-closed membrane (i.e., bladder), which is radially expanding while axially contracting when inflated with air. In the upper-limb exoskeleton, PAM/PAMs can be deployed on the robot arm, exhibiting a unidirectional structure and an antagonistic structure [14]. 
However, several challenges exist which are hindering the adoption of PAM in exoskeleton robots. First, several researchers have highlighted that the PAM system exhibits time-varying features $[22,23]$. Subsequently, as impacted by the elasticity of the bladder contained in the braided sheath and the mechanical characteristics (i.e., friction) between the bladder and the sheath combination under pressure, the PAM system is recognized as a nonlinear system [24-27]. The mentioned challenges impose difficulties on the controller design for the high-performance position tracking system [28]. In recent years, the model-based control method has become a general method in the practice control of the PAM system [29]. The theoretical model (e.g., the quasi-static physical model) and the phenomenological model (e.g., the three-element model or the high-order linear model), i.e., the compensation model, were built to express the force-contraction behavior in the PAM control system [30-32]. However, the mentioned models contain several system and controller parameters, thereby increasing the difficulty in identification and complicating the building of an accurate model. For instance, five shape parameters and two air pressure parameters are required for calibrating in the quasi-static physical model of the McKibben type PAM [26]. To solve the mentioned issues, approximation structures (e.g., the fuzzy system or artificial neural network) were purposely employed in the PAM system [22,33]. In addition, collecting accurate and sufficient data from the experiment lays a precondition for achieving the high performance of the mentioned approaches. Moreover, under the human impact, the external and uncertain disturbance dynamic model was capable of further affecting the accuracy of the model.

Given the difficulties in building accurate models, several model-free control methods can act as the alternative means for the PAM system and exoskeleton [34]. The proportional derivative (PD) and proportional integral derivative (PID) control, i.e., a useful control method, were employed in applications for their structural simplicity and fast responsive performance $[35,36]$. However, the mentioned methods fail to sufficiently control the time-varying systems or systems exhibiting high nonlinearity [37]. As information science and technology are leaping forward, model-free adaptive control (MFAC) was proposed as a different approach to address nonlinear control issues [38,39]. By complying with the theory of data-driven control, designers, regardless of whether the data originate from online or offline, are able to directly exploit the input/output information of the system. For practical engineering, MFAC can be designed by drawing on the knowledge from data processing without considering whether the mathematical model of the controlled system is explicit or implicit. Based on the above framework, MFAC combined with other nonlinear control methods is capable of improving the robustness of the system [40]. For instance, the scheme of the parameter's self-adjusting law and MFAC overcomes the parameter tuning process of the controller, and it is applicable to tracking the trajectory control in the PAM elbow robots [41]. Moreover, sliding mode control (SMC) acts as a useful nonlinear tool for dynamic systems exhibiting uncertain and bounded disturbances [42]. By combining the MFAC and the sliding mode exponential reaching law, a position tracking controller for a five-DOF upper-limb exoskeleton was proposed to assist victims of stroke in passive robot-assisted rehabilitation training [43].

The above attempts underpin the design of a position controller for the exoskeleton driven by PAM/PAMs with high robustness. In fact, the mentioned methods have not considered the accuracy of the tracking performance issue until the prescribed performance method was proposed [44]. The prescribed performance (PP) method is capable of ensuring the tracking error in a small predesigned boundary by appropriately defining the tracking error transformation function. Thus far, several studies in the literature have shifted from theoretically analyzing the problem of prescribed performance control to practical application. For instance, in [45], the prescribed performance control was employed in the MFAC, and the proposed method could theoretically converge the tracking error into a small boundary. In [38], a novel type of error transformation function was illustrated in the MFAC-SMC-PP framework, and the proposed method was applied in the shape memory alloy actuators to conduct practical operations. In [14], a second-order sliding 
mode controller integrated with the MFAC and PP method was applied for the trajectory tracking issue of the PAM upper-limb exoskeleton. However, in the mentioned controllers, the selection of parameters is dependent on the initial states, thereby indicating that the initial tracking error should be confined in the performance boundary [34]. A dilemma was identified on the application for the upper-limb exoskeleton. To be specific, once the rehabilitation training tasks have a break and should be restarted, it is required to detect the position information and select the parameters prior to the operation, thereby causing inconvenience in practice. In [34], a novel nonlinear control method was developed, and a tuning function was designed to modify the tracking error as a new variable type. Such an approach makes it possible to eliminate the limitation of the initial states without altering the stability exhibited by the system with the MFAC-SMC-PP control framework.

Inspired by the mentioned observations, this study is focused on the PP method in the tracking trajectory issue of the upper-limb exoskeleton. It primarily discusses a new MFAC-SMC-PP control method to address the initial position that does not meet the performance boundary condition, which often occurs during the restart of the exoskeleton. Unlike the original MFAC-SMC-PP control framework, a tuning function, which can be exploited to overcome the technical difficulty of the initial tracking error limitation in prescribed performance method, is introduced into the proposed control method to manipulate a one-DOF upper-limb exoskeleton driven by PAMs. On that basis, this study attempted to mimic passive rehabilitation training. Moreover, the proposed method is not determined by the dynamic model parameters, whereas it merely relies on the input and output data. Furthermore, the second-order sliding mode controller was employed to reduce the tracking error of the exoskeleton based on an unknown nonlinear dynamic and external disturbances.

The structure of this study is illustrated below. In the next section, the mechanical structure of the exoskeleton system is detailed. In Section 3, the proposed control method is presented. In Section 4, the results of several simulations and experiments are provided. Finally, in Section 5, the conclusion is drawn.

\section{One-DOF Upper-Limb Exoskeleton}

Figure 1 illustrates the structure of the one-DOF upper-limb exoskeleton, which is designed as a humanoid arm structure. It was adopted to help a stroke victim follow the joint reference trajectory of the elbow reciprocating motion (i.e., flexible/extension motion) during passive rehabilitation training. The exoskeleton is comprised of two components, i.e., a rigid robot arm (made of aluminum alloy) and a soft shoulder pad (made of dacron).

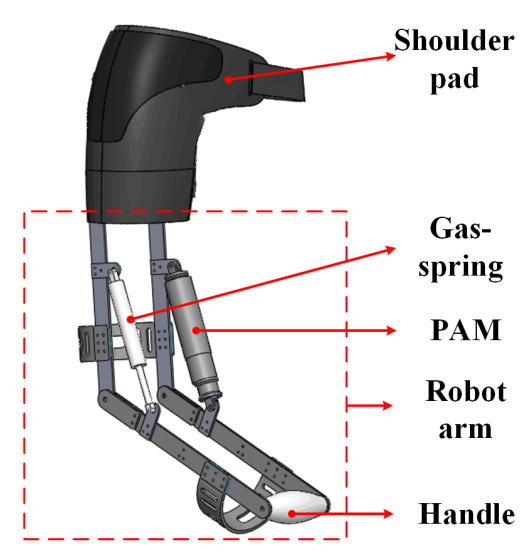

Figure 1. One-DOF upper-limb exoskeleton.

The whole robot arm is made of aluminum alloy with a mass of $1.02 \mathrm{~kg}$ in total, thereby complying with the current lightweight upper-limb exoskeleton requirements. One piece of Mckibben type PAM (i.e., length: $265 \mathrm{~mm}$, waving angle: 30 degrees, and diameter: $32 \mathrm{~mm}$, mass: $0.2 \mathrm{~kg}$ ) as the joint actuator is installed on the inner side of the robot arm to help 
users achieve elbow flexible/extension movement. For safety, the inner air pressure of the PAM ranges from 0 to 4 bars. From the existing experiment, the maximal contraction force generated from the PAM exceeded $100 \mathrm{~N}$ [14]. According to the reference, the force that the PAM used in an upper-limb exoskeleton (named RUPERT) can generate is approximately $269 \mathrm{~N}$ [15]. Meanwhile, a piece of FESTO-branded PAM can generate more than $500 \mathrm{~N}$ of pulling force with $0-5$ bar of air pressure [32].

Unlike other upper-limb exoskeletons driven by PAM, a passive mechanism is set at the elbow joint. A gas spring (internal air pressure: $0.1 \mathrm{MPa}$, stiffness: $0.26 \mathrm{~N} / \mathrm{mm}$ ), acting as an energy storage element, is installed on the outer side of the robot arm. During the elbow extension, the gas spring is capable of releasing its energy and pulling the robot arm away. At the end of the robot arm, a PLA handle is made by a 3D printer. Subsequently, a shoulder pad, which can tightly wrap around the human shoulder, is exploited to anchor the robot arm to the human shoulder. The shoulder pad primarily aims to simplify the multi-motion pair composite structure of the shoulder, thereby improving the fitness of the human shoulder with the exoskeleton and stabilizing the human acromion.

\section{Model-Free Adaptive Control with Prescribed Performance Method}

\subsection{MFAC-SMC-PP Control Framework}

The principle of MFAC-SMC-PP has been extensively studied [44,45]. The vital idea refers to finding a required function (termed error transformation function) to develop an equivalent system with several virtual variables and adopt a model-free adaptive control method to track the reference trajectory. Moreover, the SMC is used to reduce the influence of the controller under unknown disturbance [40]. In the MFAC-SMC-PP control framework, the tracking error can be limited to a small predefined boundary designed by the user without any model parameters' information. As shown in Figure 2, the prescribed performance control block can transform the tracking error into a new variable $z(k)$ with boundary information. Then, the pseudo-partial derivative estimation block estimates the pseudo partial derivative value (i.e., $\hat{\Phi}_{12}(k)$ ) according to the historical input (i.e., $u(k-1)$ ) and output (i.e., $y(k-1)$ ) of the system for the MFAC-SMC block. Finally, the MFAC-SMC block calculates the control law (i.e., $\tau(k)$ ) and sends it into the control plant (i.e., one-DOF upper-limb exoskeleton).

Assuming a discrete-time nonlinear system is considered as

$$
y(k+1)=f\left(u(k), \cdots, u\left(k-d_{u}\right), y(k), \cdots, y\left(k-d_{y}\right)\right)
$$

where $u(k)$ and $y(k)$ denote the input and output of the system at time instant $k$, respectively, $d_{u}$ denotes the length of the input, and $d_{y}$ denotes the system order.

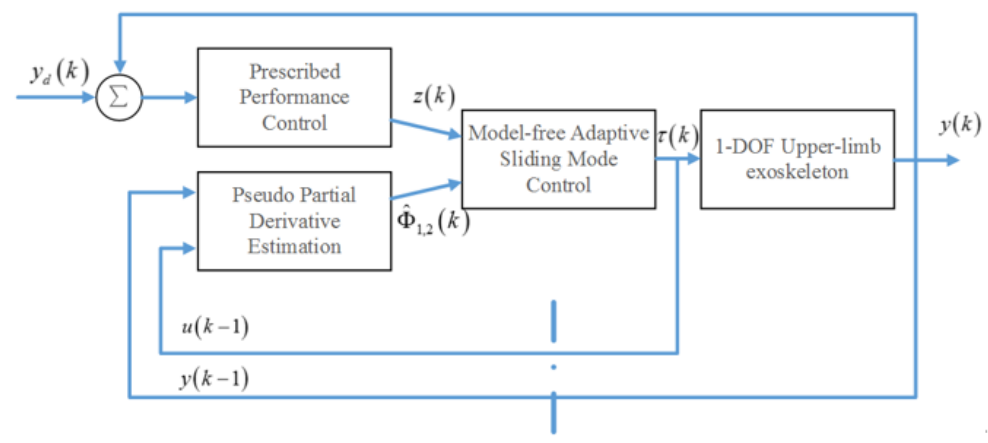

Figure 2. The original MFAC-SMC-PPC control framework. 
There are two assumptions:

Assumption 1. The partial differentials of the function of $f(\cdot)$ related to the system inputs $u(k), \cdots, u\left(k-d_{u}\right)$ are successive [38].

Assumption 2. The system in Equation (1) satisfies the generalized Lipschitz [40].

By complying with the MFAC method, if the above system satisfies the following Assumptions 1 and 2, the system is expressed as Equation (2):

$$
y(k+1)=y(k)+\Delta y(k) \Phi_{1}(k)+\Delta u(k) \Phi_{2}(k)
$$

According to the reference, an estimation approach can be exploited to determine $\Phi_{1}(k)$ and $\Phi_{2}(k)$ below [45]:

$$
\begin{aligned}
& \hat{\Phi}_{1}(k)=\hat{\Phi}_{1}(k)+\frac{\eta(\Delta y(k)-g(k-1)) \Delta y(k-1)}{l(k-1)+\mu} \\
& \hat{\Phi}_{2}(k)=\hat{\Phi}_{2}(k)+\frac{\eta(\Delta y(k)-g(k-1)) \Delta u(k-1)}{l(k-1)+\mu}
\end{aligned}
$$

where $\mu$ is adopted to prevent the generation of singular values, $\eta>0$ is a scale constant, $g(k-1)=\Delta y(k-1) \hat{\Phi}_{1}(k-1)+\Delta u(k-1) \hat{\Phi}_{2}(k-1)$, and $l(k-1)=\Delta y^{2}(k-1)+$ $\Delta u^{2}(k-1)$.

Given the modeling uncertainties and boundedness disturbance $|f(k)|<\Gamma_{1}$ (e.g., friction force/torque) [43], Equation (2) can be rewritten as

$$
y(k+1)=y(k)+\Delta y(k) \Phi_{1}(k)+\Delta u(k) \Phi_{2}(k)+f(k)
$$

To address the tracking performance issue, a reference trajectory is defined as $y_{d}(k)$. Then, the tracking error is defined as $e(k)=y(k)-y_{d}(k)$ [40]. According to the prescribed performance method [10], a convergent bounded function-termed performance function-is defined in Equation (6). It represents the boundary of the system-tracking error. The prescribed performance can be expressed as $-\rho(k)<e(k)<\rho(k)$ :

$$
\rho(k+1)=(1-v) \rho(k)+v \rho_{\infty}
$$

where $0<v s .<1$ denotes the convergence ratioand $\rho_{\infty}>0$ is defined as a positive constant. Both of these should be predefined by the user.

To solve the restricted tracking error, a strictly increasing function $S(\cdot)$, for the error transformation function, is presented as

$$
S(z)=\frac{\exp (-z)-\exp (z)}{\exp (-z)+\exp (z)}
$$

where $z$ denotes the visual state variable. It satisfies:

$$
e(k)=\rho(k) S(z)
$$

By substituting Equation (7) into Equation (8), it yields:

$$
z(k)=\frac{1}{2} \ln \left(\frac{\rho(k)+e(k)}{\rho(k)-e(k)}\right)
$$

In the framework, the SMC is an effective method of improving the tracking accuracy of the nonlinear system [36]. Accordingly, the sliding mode is defined as a proportion integration type:

$$
s(k)=z(k)+K E(k-1)
$$


where $E(k)=E(k-1)+z(k), K$ denotes the integral parameter.

Since $z(k+1)=(1-K) z(k)$, according to Equation (9), it yields:

$$
\frac{1}{2} \ln \left(\frac{\rho(k+1)+e(k+1)}{\rho(k+1)-e(k+1)}\right)=(1-K) z(k)
$$

Subsequently, a virtual control variable $\varsigma$ is set as:

$$
\varsigma(k)=\exp (2(1-K) z(k))
$$

At time instant $k+1$, the tracking trajectory error $e(k+1)=y(k+1)-y_{d}(k+1)$ is rewritten as

$$
\begin{gathered}
e(k+1)=e(k)+\Phi_{1}(k) \Delta y(k)+\Phi_{2}(k) \Delta u(k)-\Delta y_{d}(k+1) \\
\Delta y_{d}(k+1)=y_{d}(k+1)-y_{d}(k)
\end{gathered}
$$

By substituting Equation (12) into Equation (13), the control law is expressed as $u(k)=u(k-1)+u_{M F A C}(k)+u_{S M C}(k):$

$$
u_{M F A C}(k)=-\frac{1-\xi(k)}{(1+\xi(k)) \hat{\Phi}_{2}(k)} \rho(k+1)+\frac{1}{\hat{\Phi}_{2}(k)}\left(\Delta y_{d}(k+1)-e(k)-\hat{\Phi}_{1}(k) \Delta y(k)\right)
$$

The control law of $u_{S M C}(k)$ is adopted to offset the possible impact of $f(k)$ in Equation (5). In this study, a second-order sliding mode control built by the super-twisting algorithm was employed to mitigate the chattering phenomenon of the controller [46]:

$$
u_{S M}(k)=-\frac{\Gamma_{1}}{\Phi_{2}}\left(\operatorname{sign}(s(k))+\Gamma_{2}|s(k)|^{0.5} \operatorname{sign}(s(k))\right)
$$

where $\Gamma_{1} \geq|f(k)|, \Gamma_{2}>0$.

\subsection{Dynamic Description and Controller Design}

Since the one-DOF upper-limb exoskeleton covers one active joint on the elbow in order to assist human elbow extension and flexion movement, the dynamic system is simplified as a one-DOF rigid link model (as shown in Figure 3). It can be expressed as

$$
M \ddot{q}+C \dot{q}+G+\tau_{d}=\tau
$$

where $\tau_{d}$ denotes the sum of the external disturbance and dynamic model uncertainty; $M \ddot{q}$ is the inertia torque; $C \dot{q}$ represents the joint friction torque; $G$ represents the gravitation torque; $\dot{q}$ and $\ddot{q}$ denote the first derivative and second derivative of the joint position $q$, respectively.

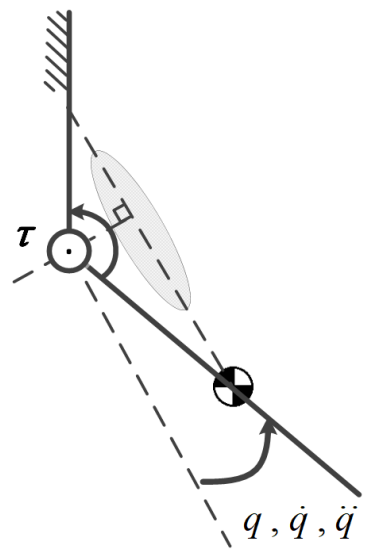

Figure 3. Rigid link model. 
According to the reference [43], the state form of the dynamic system is discretized as

$$
\begin{gathered}
\ddot{q}(k)=\frac{\tau(k)-D(k)}{M} \\
D(k)=G(k)+C \dot{q}(k)+\tau_{d}(k)
\end{gathered}
$$

Subsequently, $T$ is set as the sample period of the system. $\tau(k)$ denotes the input signal $u(k) . \Delta q(k)=q(k)-q(k-1)$ represents the output signal $u(k)$. Then, Equation (18) can be transformed into Equation (20):

$$
y(k+1)=y(k)+\frac{T^{2} u(k)}{M}+\Delta f(k)
$$

where $\Delta f(k)=-T^{2} D(k) / M$.

As previously mentioned, $y(k+1)$ thereby demonstrates that the system is a generalized Lipschitz system, with respect to the output signal $y(k)$ and the input signal $u(k)$, and the controller can be designed to be obeyed in the MFAC-SMC-PP control framework. Hence, the dynamic of the one-DOF upper-limb exoskeleton can be translated to the system described in Equation (5).

However, the upper-limb exoskeleton may restart according to the user's mind, and the tracking trajectory control should also be reset. In this situation, the time constant $k$ can be easily reset to 0 , but the joint position $y(k)$ may not be easily reset to $y(0)$. Then, the original MFAC-SMC-PP controller may not work since the initial value of $e(0)$ may escape from the boundary. Hence, a novel prescribed performance method is introduced below. Inconsistently with the original MFAC-SMC-PP control framework, a tuning function is adopted to modify the tracking error $e(k)$ into a novel variable $b(e)$, thereby demonstrating that the prescribed performance controller is not correlated with the initial error.

As highlighted by the related literature [24,34], the tuning function is defined as

$$
\begin{aligned}
& b(e)=\varphi(t) e(t) \\
& \varphi(t)=\left\{\begin{array}{cc}
\sin \left(\frac{\pi t}{2 t_{\text {in }}}\right) & t<k_{\text {in }} \\
1 & \text { other }
\end{array}\right.
\end{aligned}
$$

where $k_{\text {in }}$ denotes a constant which should consider the maximal output of actuators and the convergence time designed by the user [34].

Then, the visual state variable of $z$ can be determined as

$$
b(e)=\rho(k) S(z)
$$

In the proposed method, the prescribed performance function satisfies:

$$
\rho(k)=\rho(k+1)=\rho
$$

where $\rho$ is a constant which can replace $\rho_{\infty}$ from Equation (6). The tracking error can be bounded as

$$
-\rho<e(k)<\rho
$$

Selecting the identical strictly increasing function of $S(z)$, Equation (9) can be rewritten as

$$
z(k)=\frac{1}{2} \ln \left(\frac{\rho+b(e(k))}{\rho-b(e(k))}\right)
$$

By complying with the designing order of the MFAC-SMC-PP control framework, the identical sliding mode variable of $s(k)$ and the equivalent reaching law are defined. Equation (11) can be rewritten as 


$$
\begin{aligned}
& \frac{1}{2} \ln \left(\frac{\rho+b(e(k+1))}{\rho-b(e(k+1))}\right)=(1-K) z(k) \\
& \frac{\rho+b(e(k+1))}{\rho-b(e(k+1))}=\exp (2(1-K) z(k))
\end{aligned}
$$

The virtual control variable $\varsigma(k)$ is represented as

$$
\varsigma(k)=\frac{\rho+b(e(k+1))}{\rho-b(e(k+1))}
$$

By substituting Equation (28) into Equation (27), the function can be simplified to:

$$
e(k+1)=-\frac{1-\varsigma(k)}{(1+\varsigma(k)) \varphi(k+1)} \rho
$$

Thus, the control law in Equation (15) is redesigned as

$$
u_{M F A C}(k)=-\frac{1-\xi(k)}{(1+\xi(k)) \varphi(k+1) \hat{\Phi}_{2}(k)} \rho+\frac{1}{\hat{\Phi}_{2}(k)}\left(\Delta y_{d}(k+1)-e(k)-\hat{\Phi}_{1}(k) \Delta y(k)\right)
$$

Finally, the proposed control method is depicted in Figure 4 to provide more insights.

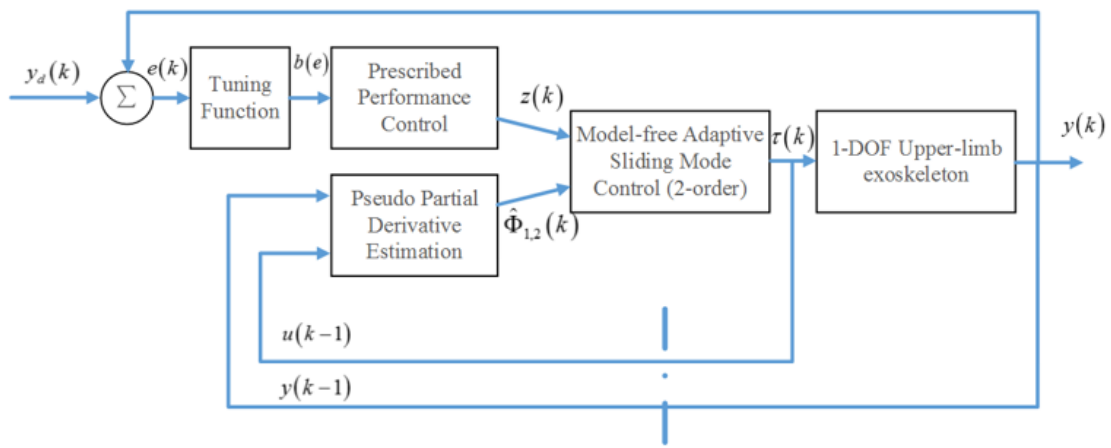

Figure 4. MFAC-SMC-PPC control framework with a tuning function.

\subsection{Stability Analysis}

According to reference [14], the Lyapunov function was selected to be $V=s^{2} / 2$. After differentiation and linearization, it yields:

$$
\dot{V} \rightarrow \frac{(s(k)-s(k-1))}{T} s(k)=\frac{1}{T} s(k) \Delta s(k)
$$

Given $T>0, \dot{V}$ and $s(k) \Delta s(k)$ are of same sign. Since $\Delta s(k)=s(k+1)-s(k)$, it yields:

$$
\Delta s(k)=z(k+1)-(1-K) z(k)
$$

By substituting Equations (25) and (29) into (32), it also yields:

$$
\Delta s(k)=\frac{1}{2} \ln \left(\frac{\rho+\varphi(k+1) e(k+1)}{\varsigma(k) \rho-\varsigma(k) \varphi(k+1) e(k+1)}\right)
$$

Using Equations (16), (30) and (33), $\Delta s(k)$ is denoted by 


$$
\Delta s(k)=\frac{1}{2} \ln \left(\frac{\frac{2 \zeta(k)}{1+\zeta(k)} \rho+\left(\Delta f(k)-\Gamma_{1}\left(\operatorname{sign}(s(k))+\Gamma_{2}|s(k)|^{0.5} \operatorname{sign}(s(k))\right)\right)}{\varsigma(k)\left(\frac{2}{1+\varsigma(k)} \rho-\left(\Delta f(k)-\Gamma_{1}\left(\operatorname{sign}(s(k))+\Gamma_{2}|s(k)|^{0.5} \operatorname{sign}(s(k))\right)\right)\right)}\right)
$$

From references [38,45], it yields:

$$
\begin{aligned}
& v_{a}=2 \rho \\
& v_{b, k}=\frac{1+\varsigma(k)}{\varsigma(k)} \\
& v_{c, k}=1+\varsigma(k)
\end{aligned}
$$

Since $\varsigma(k)$ refers to an exponential function (i.e., $\zeta(k)>0$ ), $v_{b, k}$ and $v_{c, k}$ are positive. Moreover, $v_{a}>0$ is a positive constant. Then, the stability analysis is expressed below.

Step 1 . If $s(k)>0, \operatorname{sign}(s(k))=1 . \Delta s(k)$ can be denoted by

$$
\Delta s(k)=\frac{1}{2} \ln \left(\frac{v_{a}+v_{b, k}\left(\Delta f(k)-\Gamma_{1}\left(1+\Gamma_{2}|s(k)|^{0.5}\right)\right)}{v_{a}-v_{c, k}\left(\Delta f(k)-\Gamma_{1}\left(1+\Gamma_{2}|s(k)|^{0.5}\right)\right)}\right)
$$

Since $|\Delta f(k)| \leq \Gamma_{1}$ and $\Gamma_{2}>0$, it yields $\Gamma_{1}\left(1+\Gamma_{2}|s(k)|^{0.5}\right)>\Gamma_{1} \geq|\Delta f(k)|$. Then, $v_{a}+v_{b, k}\left(\Delta f(k)-\Gamma_{1}\left(1+\Gamma_{2}|s(k)|^{0.5}\right)\right)<v_{a} . v_{a}<v_{a}-v_{c, k}\left(\Delta f(k)-\Gamma_{1}\left(1+\Gamma_{2}|s(k)|^{0.5}\right)\right)$. This can yield $\Delta s(k)<0$.

Step 2. If $s(k)<0, \operatorname{sign}(s(k))=-1 . \Delta s(k)$ can be denoted by

$$
\Delta s(k)=\frac{1}{2} \ln \left(\frac{v_{a, k}+v_{b, k}\left(\Delta f(k)+\Gamma_{1}\left(1+\Gamma_{2}|s(k)|^{0.5}\right)\right)}{v_{a, k}-v_{c, k}\left(\Delta f(k)+\Gamma_{1}\left(1+\Gamma_{2}|s(k)|^{0.5}\right)\right)}\right)
$$

This yields that $v_{a, k}-v_{c, k}\left(\Delta f(k)+\Gamma_{1}\left(1+\Gamma_{2}|s(k)|^{0.5}\right)\right)<v_{a, k}$. Then, we can obtain that $v_{a, k}<v_{a, k}+v_{b, k}\left(\Delta f(k)+\Gamma_{1}\left(1+\Gamma_{2}|s(k)|^{0.5}\right)\right)$, which means $\Delta s(k)>0$.

Accordingly, whether $s(k)>0$ or $s(k)<0$, we can obtain that $s(k) \Delta s(k)<0$ and that the proposed controller is stable.

Finally, the convergence analysis of the tracking error is presented below. Since the tracking error does not enter the predesigned boundary before $k_{i n}$, the convergence analysis was discussed for $t>k_{\text {in }}$.

Selecting a time instant of $k_{t}$ in the control process and defining $k_{t} \geq k_{i n}$, the convergence analysis process can be expressed step by step.

Step 1. Assuming $s\left(k_{t}-1\right)>0$. In the stable domain, it yields that $s\left(k_{t}-1\right) \Delta s\left(k_{t}-1\right)$ $<0$, and then $s\left(k_{t}\right)<s\left(k_{t}-1\right)$. According to Equation (32), it yields:

$$
s\left(k_{t}\right)-s\left(k_{t}-1\right)=z\left(k_{t}\right)+(K-1) \mathrm{z}\left(k_{t}-1\right) \leq 0
$$

As impacted by $z\left(k_{t}-1\right)=0.5 \ln \left(\left(\rho+e\left(k_{t}-1\right)\right) /\left(\rho-e\left(k_{t}-1\right)\right)\right),-\rho<e\left(k_{t}-1\right)<\rho$, we can see that $z\left(k_{t}-1\right)>0$.

To ensure Equation (24), $z\left(k_{t}\right)<0$ and $\ln \left(\left(\rho+e\left(k_{t}\right)\right) /\left(\rho-e\left(k_{t}\right)\right)\right)<0$. This yields that:

$$
\begin{aligned}
& 0<\frac{\rho+e\left(k_{t}\right)}{\rho-e\left(k_{t}\right)}<1 \\
& \rho+e\left(k_{t}\right)>0 \\
& \rho-e\left(k_{t}\right)>0
\end{aligned}
$$

Since $-\rho<e\left(k_{t}\right)<0$, the tracking error was successfully bounded in the prescribed performance function under the condition. 
Step 2. Assume that $s\left(k_{t}-1\right)<0$. In the stable domain, this yields that $\left(s_{k_{t}}-1\right)-$ $\left(\Delta s k_{t-1}-1\right)>0$ and $s^{2}\left(k_{t}\right) / 2<s^{2}\left(k_{t-1}\right) / 2$. Then, we can obtain $s\left(k_{t}-1\right)<s\left(k_{t}\right)<$ $\left.-s\left(k_{t}-1\right)\right)$.

A similar deduction is presented as in Equation (40). As impacted by $z\left(k_{t}\right)=$ $0.5 \ln ((\rho+e(k)) /(\rho-e(k)))$, it yields $-\rho<e(k)<\rho$. Thus, also indicates that the tracking error converged:

$$
\begin{gathered}
s\left(k_{t}\right)+(K-1) \mathrm{z}\left(k_{t}-1\right)-z\left(k_{t}\right) \leq s\left(k_{t}\right) \leq z\left(k_{t}\right)-(K-1) \mathrm{z}\left(k_{t}-1\right)-s\left(k_{t}\right) \\
0<(K-1) \mathrm{z}\left(k_{t}-1\right) \leq z\left(k_{t}\right)
\end{gathered}
$$

\section{Simulation and Experiment}

\subsection{Simulation}

In this section, two simulation cases were built to verify the validity of the obtained controller design method in the exoskeleton robot. By employing a one-DOF PAM exoskeleton system as the control plant in Equation (41), the relative parameters were obtained from the relative reference [47]. The reference trajectory was selected as a sinusoidal curve given by Equation (42):

$$
\begin{gathered}
0.75 \ddot{q}+0.17 \dot{q}+0.84 \sin (q)+0.05 \operatorname{sign}(q)=\tau \\
y_{d}=30 \sin (0.2 \pi t)
\end{gathered}
$$

In the first group of the simulation, the initial joint positions from the two controllers (the original MFAC-SMC-PP method and the proposed method) were set to 0 degrees. The proposed controller parameters were selected as $\eta=10, \mu=0.6, k_{\text {in }}=5 \mathrm{~s}, \rho=0.5$ degrees, $K=0.5, \Phi_{1}(0)=\Phi_{2}(0)=0.75, \Gamma_{1}=0.05$ and $\Gamma_{2}=0.01$. In addition, the original MFAC-SMC-PP controller was designed as the comparison method, and its controller parameters are selected to be identical to the proposed controller except for the prescribed performance function (i.e., $\rho_{0}=5, v=0.05$, and $\rho_{\infty}=0.05$ ). Moreover, to further demonstrate that the proposed method was relieved from the requirement of the initial tracking error, the initial joint position was set to -10 degrees in the second group. The tracking trajectory and controller parameters remain.

The results were obtained from the discussed simulations (in Figures 5-10). In group 1, the system output and tracking error generated from the proposed method are consistent with the results achieved using the original method (i.e., the root mean squared error: 0.15 degrees with the original method and 0.18 degrees with the proposed method; the maximal absolute value of the tracking error: 3.81 degrees with the original method and 3.25 degrees with the proposed method). Since the two methods cover prescribed performance functions, the tracking error is finally converged to the upper boundary and the lower boundary (i.e., $(-0.5,0.5)$ degrees). Furthermore, the tracking error obtained by the proposed method enters the boundary in finite time (nearly $0.05 \mathrm{~s}$ ), thereby satisfying the time requirement (i.e., $0.05<k_{\text {in }}$ ).

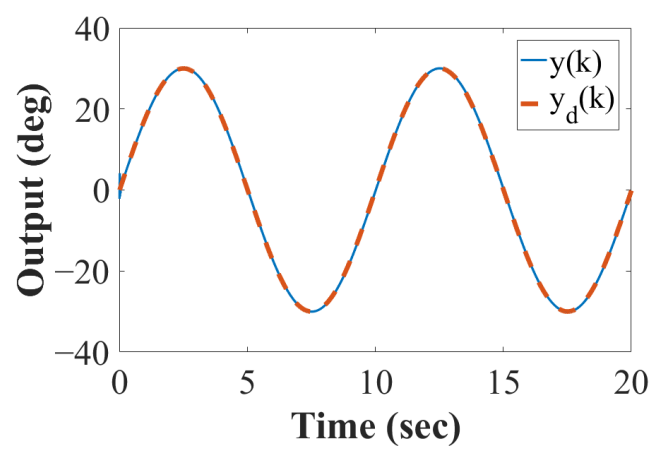

Figure 5. Output signals of the simulation in group 1 (original method). 


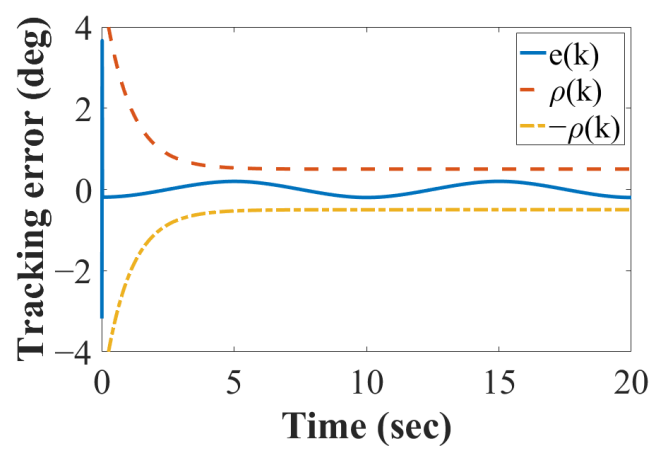

Figure 6. Tracking errors of the simulation in group 1 (original method).

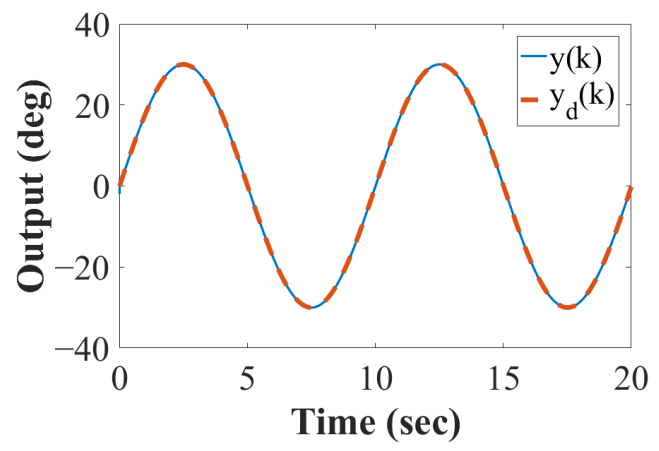

Figure 7. Output signals of the simulation in group 1 (proposed method).

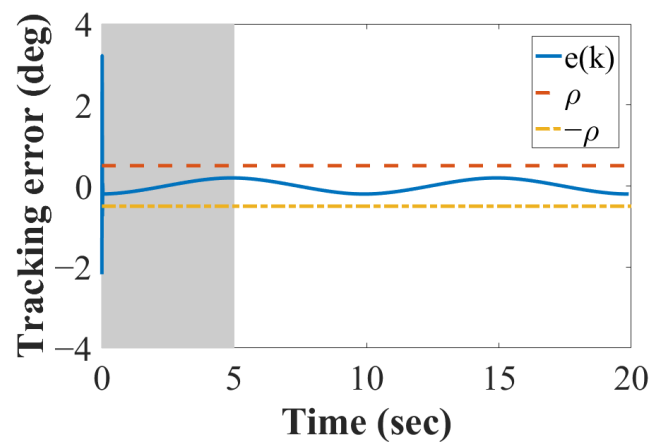

Figure 8. Tracking errors of the simulation in group 1 (proposed method).

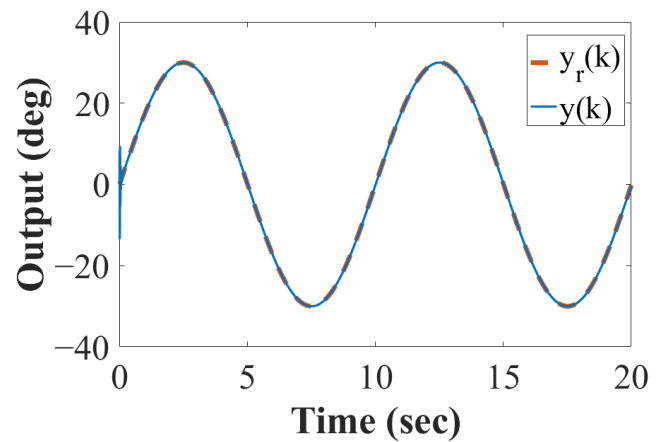

Figure 9. Output signals of the simulation in group 2 (proposed method).

Moreover, when the original method is applied, the initial errors should be confined in the predesigned boundary. Once a novel tracking task is assigned or the system needs to restart with an unknown position, certain design parameters of the controllers should be reselected-which is inconvenient for the user. For instance, in group 2, the initial position was set to -10 degrees. After several adjustments, the tracking error converged into the predesigned boundary before $k_{i n}$ in the proposed method, as shown in Figure 10 
(i.e., the gray area). The root mean squared of the tracking error is equal to 0.41 degrees, and the maximal absolute value of the tracking error is 10 degrees (i.e., $|e(0)|)$. However, since $|e(0)|>\left|\rho_{0}\right|$, the original control method cannot work until the parameters of the prescribed performance function are reselected (particularly in $\rho_{0}$ and $v$ ). Based on the discussed results, the joint position tracking trajectory task for the upper-limb exoskeleton can be fulfilled with the proposed method.

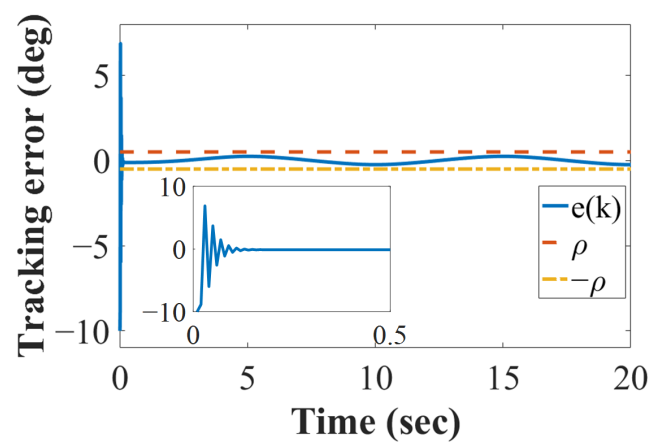

Figure 10. Tracking errors of the simulation in group 2 (proposed method).

\subsection{Experiment}

The existing simulation verifies the ability of the proposed control method to track the joint position trajectory in a virtual environment. To further illustrate the performance of the proposed controller applied in the upper-limb exoskeleton robot, an experiment was designed to mimic the robot-assisted passive rehabilitation task on the human elbow joint. First, the experimental setups were introduced. The whole equipment involved in the experiment is depicted in Figure 11. The proposed control method was programmed in a host with an Intel Core i7-4790 processor and 16 GB RAM. The execution rate can take up $1 \mathrm{KHz}$ by running the Simulink real-time programs (Matlab2016b, Mathworks Inc., Natick, MA, USA). For safety, a manikin arm was installed on the exoskeleton instead of on the actual arm during the experiment. In the sensor system, a force sensor (BJM-3, JN sensor Inc., Bengbu, Anhui, China) was installed on the exoskeleton to detect the actual joint drive torque determined by the joint position, which achieves the torque-loop of the exoskeleton system (with a programmed PI controller). Subsequently, the two inertial measurement units (WT61PC, Witmotion Inc, Beijing, China) were adopted to measure the actual joint position. The mentioned signals were acquired with a microcontroller (Arduino Mega2560, Hesai Technology Inc., Shenzhen, China) and then transmitted to the host at a communication frequency of $100 \mathrm{~Hz}$. In the meantime, another microcontroller is exploited to send the control signal to the printed circuit board to drive the two high-speed valves (MHE2-MS1H-3/20-M7, FESTO AG \& Co. KG, Esslingen Berkheim, Germany). Then, gas from the air source flows through the air filter regulator via the high-speed valves and finally passes into the PAM.

The experimental process is illustrated in Figure 12. A mimic robot-assisted passive rehabilitation training environment is set with a certain sense of reality. By complying with the proposed control method, the upper-limb exoskeleton performs a periodic flexion and extension movement (with 25 degrees of amplitude) on the elbow joint (as shown in Figure 13). The training trajectory was set as a Fourier function, as expressed in Equation (43), which applies to the human joint training trajectory [14]:

$$
q_{d}(k)=15.36-12.67 \cos (0.5 k T)-1.23 \sin (0.5 k T)-2.49 \cos (k T)-0.48 \sin (k T)
$$

The parameters of the proposed controller were selected as $\eta=30, \mu=0.6, \Phi_{1}(0)=0.95$, $\Phi_{2}(0)=0.95, t_{i n}=5 \mathrm{~s}, \rho=2$ degrees, $K=1.5, \Gamma_{1}=0.05$, and $\Gamma_{2}=0.001$. Then, the initial elbow position remained distant from the reference value with several instances of human intervention (i.e., $y(0)=10$ ) using the proposed controller. Moreover, an original MFACSMC-PP controller was used to track the same reference trajectory, except for $y(0)=0$. 
The parameters of the prescribed performance function were selected as $v=0.05, \rho(0)=5$, and $\rho=2.5$. Other parameters are as same as the proposed controller. The experimental results are presented as follows.

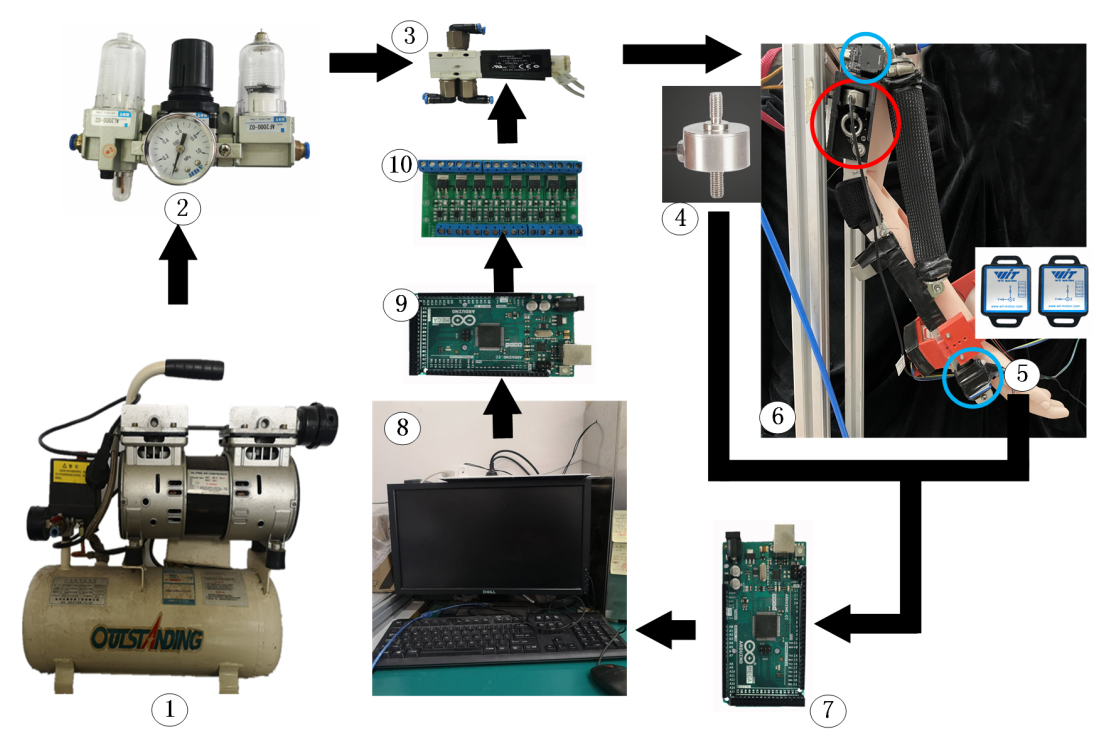

Figure 11. Experimental setups: one-air source; two-air filter regulator; three-high-speed valve; fourforce sensor (used to calculate the joint torque); five-inertial measurement unit (IMU); six-one-DOF exoskeleton and a manikin arm; seven-microcontroller(Arduino Mega 2560, employed for signal acquisition); eight-host; nine-microcontroller (Arduino Mega 2560, used for transmitting the control signal); ten-printed circuit board (adopted to drive the high-speed valve).

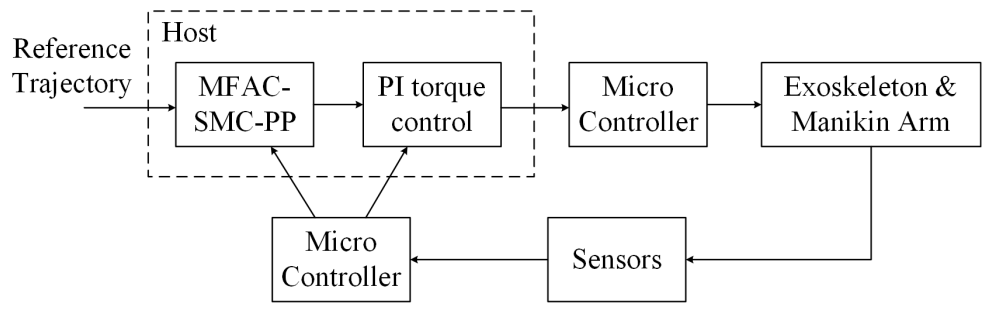

Figure 12. Block diagram of the experimental process.

According to Figure 14, by using the proposed method, the actual joint angular position is capable of effectively tracking the reference trajectory in the rehabilitation experiment. Meanwhile, the actual joint position error $q-q_{d}$ is depicted. From Figure 15, the error curve can fall into two parts by $t=k_{i n}$, i.e., the curve in the gray area and the white area. In the first part, the error curve is progressively regulated and finally enters the predesigned boundary as impacted by the tuning function. At $t=0.9 \mathrm{~s}$, the joint position error of the exoskeleton is equal to -2.53 degrees (i.e., the maximal value of the negative error). As indicated in the second part, the joint position error of the exoskeleton never escapes the boundary of $(-\rho, \rho)$. From the results, the root mean square of the joint position error is equal to 1.06 degrees. Additionally, the maximal absolute value of the steady-state error is 1.91 degrees, which is better than the correlative studies about the PAM exoskeleton systems ( \pm 2 degrees in PAM-functional electrical stimulation (FES) rehabilitation exoskeleton [15], 3.27 degrees in a PAM-driven exoskeleton [33], and 2.29 degrees in the previous study of the authors [14]). Moreover, by using the original method, the tracking error converges by 2.5 degrees within $5 \mathrm{~s}$ and the root mean square value is 1.79 degrees. These values are both slightly higher than the proposed method. Then, the original method can only work when the initial error is within the boundary defined by the prescribed performance function. The above results can indicate the high tracking performance of the proposed method in the control of exoskeleton systems. It is noteworthy that the proposed 
control method can ensure the ultimate tracking accuracy preassigned by the users while it has no requirements in the dynamic model or experience knowledge, and it even has no information regarding the initial tracking error. Hence, the proposed control method has potential application in passive robot-assisted rehabilitation.

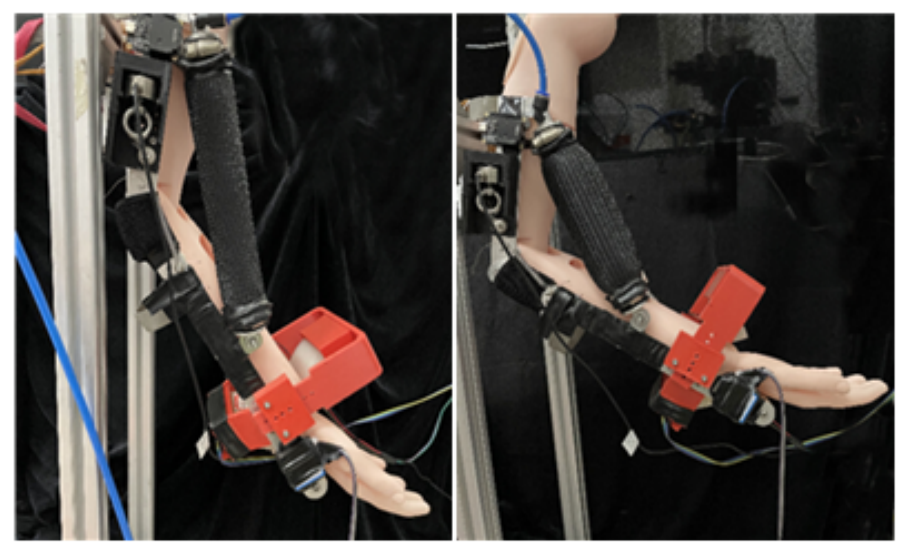

Figure 13. Passive robot-assisted rehabilitation.

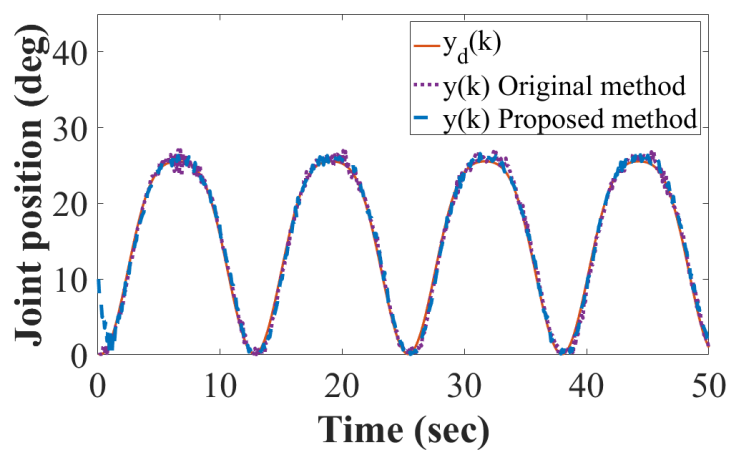

Figure 14. Experimental result (joint position).

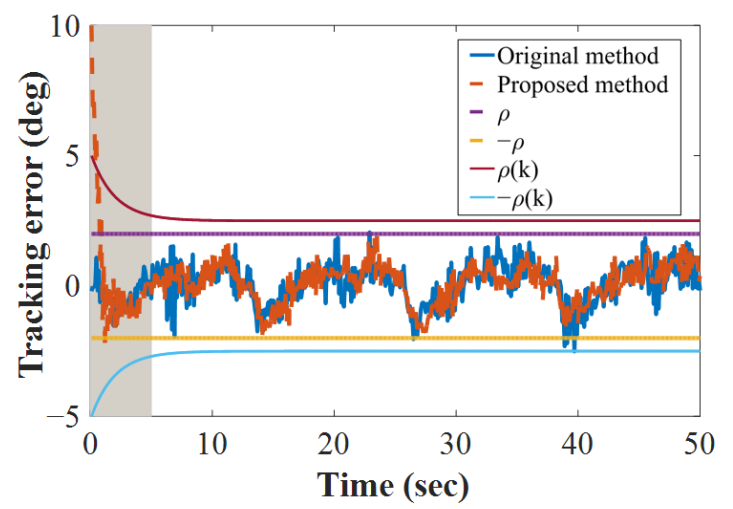

Figure 15. Experimental result (tracking error).

\section{Conclusions}

To summarize, this study proposed a novel MFAC-SMC-PP control method for the one-DOF upper-limb exoskeleton robot driven by the PAM to fulfill passive robot-assisted rehabilitation tasks when the initial tracking error is out of the boundary. According to the proposed method, a tuning function and prescribed performance function are exploited to transform the tracking error into a novel type of variable. This changes the framework of the original MFAC-SMC-PP, thereby causing the controller design to not be correlated with the initial conditions. Moreover, based on the MFAC framework, the proposed control method can evade the need for auxiliary approximating structures and model calibration. 
The experimental results show that the tracking errors of the proposed control steadystate error can converge into the predesigned boundary, and the root mean squared of the tracking error is equal to 1.06 degrees. Subsequent work will be dedicated (but not limited) to improving a safer exoskeleton for rehabilitation training tasks and extending the developed method to a more complex MIMO system for position tracking tasks (e.g., a multi-DOF exoskeleton system). To be specific, the presented controller will be integrated with the virtual impedance control method for the exoskeleton system to evaluate human movement in active robot-assisted rehabilitation.

Author Contributions: Literature search, program, data collection and writing-original draft, Z.Z.; data interpretation, L.H., J.X. and H.J.; methodology, Z.Z.; experiment and editing, Z.Z. and H.Z. All authors have read and agreed to the published version of the manuscript.

Funding: This work was supported by the National Natural Science Foundation of China under grant number 62073063.

Institutional Review Board Statement: Not applicable.

Informed Consent Statement: Not applicable.

Data Availability Statement: Data is contained within the article.

Acknowledgments: The authors thank Xifeng Gao and Mingfang Liu for their helpful advice and comments on this study.

Conflicts of Interest: The authors declare no conflict of interest.

\section{References}

1. Joel, C.P.; Jacob, R.; Stephen, B. Upper-Limb Powered Exoskeleton Design. IEEE/ASME Trans. Mechatron. 2007, 12, 408-417.

2. Joudzadeh, P.; Hadi, A.; Tarvirdizadeh, B.; Borooghani, D.; Alipour, K. Design and fabrication of a lower limb exoskeleton to assist in stair ascending. Ind. Robot. 2019, 46, 209-299. [CrossRef]

3. Lee, H.D.; Lee, B.K.; Kim, W.S.; Han, J.S.; Shin, K.S.; Han, C.S. Human-robot cooperation control based on a dynamic model of an upper limb exoskeleton for human power amplification. Mechatronics 2014, 24, 168-176. [CrossRef]

4. Tripathy, S.; Panicker, R.; Shrey, S.; Naik, R.; Pachpore, S.S. Voice controlled upper body exoskeleton: A development for industrial application. Int. J. Sci. Technol. Res. 2020, 9, 1032-1037.

5. Ghan, J.; Kazerooni, H. System identification for the berkeley lower extremity exoskeleton (bleex). In Proceedings of the International Conference on Robotics and Automation, Orlando, FL, USA, 15-19 May 2006; pp. 3477-3484.

6. Wang, S.; Wang, L.; Meijneke, C.; Asseldonk, E.V.; Hoellinger, T.; Cheron, G. Design and control of the mindwalker exoskeleton. IEEE Trans. Neural Syst. Rehabil. Eng. 2015, 23, 277-286. [CrossRef]

7. Lo H.S.; Sheng, Q.X. Exoskeleton robots for upper-limb rehabilitation: State of the art and future prospects. Med. Eng. Phys. 2012, 34, 261-268. [CrossRef]

8. Gopura, R.A.R.C.; Kiguchi, K. Development of a 6 DOF Exoskeleton Robot for Human Upper-Limb Motion Assist. In Proceedings of the International Conference on Information and Automation for Sustainability, Colombo, Sri Lanka, 12-14 December 2008; pp. 13-18.

9. Gupta, A.; Malley, M.K. Design of a Haptic Arm Exoskeleton for Training and Rehabilitation. IEEE/ASME Trans. Mechatron. 2006, 11, 280-289. [CrossRef]

10. Vitiello, N.; Lenzi, T.; Roccella, S.; De Rossi, S.M.M.; Cattin, E.; Giovacchini, F.; Vecchi, F.; Carrozza, M.C. NEUROExos: A Powered Elbow Exoskeleton for Physical Rehabilitation. IEEE Trans. Robot. 2013, 29, 220-235. [CrossRef]

11. Wang, X.; Li, X.; Wang, J. Modeling and Identification of the Human-Exoskeleton Interaction Dynamics for Upper Limb Rehabilitation. In Proceedings of the Chinese Intelligent Automation Conference, Beijing, China, 21 March 2015; pp. 51-60.

12. Brewer, L.; Horgan, F.; Hickey, A.; Williams, D. Stroke rehabilitation: Recent advances and future therapies. QJM Mon. J. Assoc. Phys. 2013, 106, 11-25. [CrossRef] [PubMed]

13. Xiao, F.; Gao, Y.; Wang, Y.; Zhu, Y.; Zhao, J. Design and evaluation of a 7-DOF cable-driven upper limb exoskeleton. J. Mech. Sci. Technol. 2018, 32, 855-864. [CrossRef]

14. Zhao, Z.; Hao, L.; Liu, M.; Gao, H.; Li, X. Prescribed performance model-free adaptive terminal sliding mode control for the pneumatic artificial muscles elbow exoskeleton. J. Mech. Sci. Technol. 2021, 35, 3183-3197. [CrossRef]

15. Tu, X.; Zhou, X.; Li, J.; Su, C.; He, J. Iterative learning control applied to a hybrid rehabilitation exoskeleton system powered by PAM and FES. Clust. Comput. 2017, 20, 2855-2868. [CrossRef]

16. Kalita, B.; Dwivedy, S.K. Dynamic analysis of pneumatic artificial muscle (PAM) actuator for rehabilitation with principal parametric resonance condition. Nonlinear Dyn. 2019, 97, 2271-2289. [CrossRef] 
17. Gao, X.; Sun, Y.; Hao, L.; Yang, H.; Chen, Y.; Xiang, C. A New Soft Pneumatic Elbow Pad for Joint Assistance With Application to Smart Campus. IEEE Access 2018, 6, 38967-38976. [CrossRef]

18. Noda, T.; Teramae, T.; Ugurlu, B.; Morimoto, J. Development of an upper limb exoskeleton powered via pneumatic electric hybrid actuators with bowden cable. In Proceedings of the IROS, Chicago, IL, USA, 14-18 September 2014; pp. 3573-3578.

19. Zhang, J.F.; Yang, C.J.; Chen, Y.; Zhang, Y.; Dong, Y.M. Modeling and control of a curved pneumatic muscle actuator for wearable elbow exoskeleton. Mechatronics 2008, 18, 448-457. [CrossRef]

20. Kamil, I.; Ondrej, L.; Vladislav, M. Advanced rehabilitation device based on artificial muscle actuators with neural network implementation. J. Autom. Mob. Robot. Intell. Syst. 2012, 6, 30-32.

21. Jian, H.; Tu, X.; He, J. Design and Evaluation of the RUPERT Wearable Upper Extremity Exoskeleton Robot for Clinical and In-Home Therapies. IEEE Trans. Syst. Man Cybern. Syst. 2016, 46, 926-935.

22. Anh, H.; Ahn, K.K. Identification of pneumatic artificial muscle manipulators by a MGA-based nonlinear NARX fuzzy model. Mechatronics 2009, 19, 106-133. [CrossRef]

23. Sarosi, J. Accurate positioning of pneumatic artificial muscle at different temperatures using LabVIEW based sliding mode controller. In Proceedings of the IEEE International Symposium on Applied Computational Intelligence and Informatics, Timisoara, Romania, 15-17 May 2014; pp. 85-89.

24. Gao, X.; Li, X.; Zhao, C.; Hao, L.; Xiang, C. Variable stiffness structural design of a dual-segment continuum with independent stiffness and angular position. Robot. Comput. Integr. Manuf. 2021, 67, 102000. [CrossRef]

25. Yang, H.; Zhang, Y.; Chen, Y.; Sun, Y.; Hao, L. A novel learning adaptive hysteresis inverse compensator for pneumatic artificial muscles. Smart Mater. Struct. 2019, 29, 015035. [CrossRef]

26. Chou, C.P.; Hannaford, B. Measurement and modeling of Mckibben pneumatic artificial muscles. IEEE Trans. Robot. Autom. 1996, 12, 90-102. [CrossRef]

27. Aschemann, H.; Schindele, D. Comparison of model-based approaches to the compensation of hysteresis in the force characteristic of pneumatic muscles. IEEE Trans. Ind. Electron. 2014, 61, 3620-3629. [CrossRef]

28. Vo-Minh, T.; Tjahjowidodo, T.; Ramon, H.; Brussel, H.V. A new approach to modeling hysteresis in a pneumatic artificial muscle using the maxwell-slip model. IEEE/ASME Trans. Mechatron. 2011, 16, 177-186. [CrossRef]

29. Ugurlu, B.; Forni, P.; Doppmann, C.; Sariyildiz, E.; Morimoto, J. Stable control of force, position, and stiffness for robot joints powered via pneumatic muscles. IEEE Trans. Ind. Inform. 2019, 15, 6270-6279. [CrossRef]

30. Hassan, T.; Cianchetti, M.; Moatamedi, M.; Mazzolai, B.; Laschi, C.; Dario, P. Finite-Element Modeling and Design of a Pneumatic Braided Muscle Actuator With Multifunctional Capabilities. IEEE/ASME Trans. Mechatron. 2019, 24, 109-119. [CrossRef]

31. Robinson, R.M.; Kothera, C.S.; Wereley, N.M. Nonlinear analysis of quasi-static response of pneumatic artificial muscles for agonistic and antagonistic actuation modes. In Proceedings of the SPIE Bioinspiration, Biomimetics, and Bioreplication, San Diego, CA, USA, 11-13 March 2013; pp. 796-809.

32. Wickramatunge, K.C.; Leephakpreeda, T. Study on mechanicalbehaviors of pneumatic artificial muscle. Int. J. Eng. Sci. 2010, 48, 188-198 [CrossRef]

33. Cao, Y.; Huang, J. Neural-network-based nonlinear model predictive tracking control of a pneumatic muscle actuator-driven exoskeleton. IEEE J. Autom. Sin. 2013, 7, 1478-1488. [CrossRef]

34. Gao, X.; Li, X.; Sun, Y.; Hao, L.; Yang, H.; Xiang, C. Model-free tracking control of continuum manipulators with global stability and assigned accuracy. IEEE Trans. Syst. Man Cybern. Syst. 2020,1-11. [CrossRef]

35. Liang, G.; Ye, W.; Xie, Q. PID control for the robotic exoskeleton: Application to lower extremity rehabilitation. In Proceedings of the IEEE International Conference on Mechatronics and Automation, Chengdu, China, 5-8 August 2012; pp. 2345-2350.

36. Roy, R.; Islam, M.; Rashid, M.; Mounis, S.; Ahsan, M.M.; Ahad, M.T.; Siddique, Z.; Kouzani, A.Z.; Mahmud, M.A.P. Investigation of 2DOF PID Controller for Physio-Therapeutic Application for Elbow Rehabilitation. Appl. Sci. 2021, 11, 8617. [CrossRef]

37. Zawawi, M.; Elamvazuthi, I.; Aziz, A.; Da Ud, S.A. Comparison of PID and fuzzy logic controller for DC servo motor in the development of lower extremity exoskeleton for rehabilitation. In Proceedings of the IEEE International Symposium in Robotics and Manufacturing Automation, Kuala, Lumpur, 19-21 September 2017; pp. 1-6.

38. Liu, M.; Zhao, Z.; Hao, L. Prescribed performance model-free adaptive sliding mode control of a shape memory alloy actuated system. ISA Trans. 2021, in press.

39. Hou, Z.S.; Wang, Z. From model-based control to data-driven control: Survey, classification and perspective. Inf. Sci. 2013, 235 , 3-35. [CrossRef]

40. Wang, Y.; Hou, M. Model-free adaptive integral terminal sliding mode predictive control for a class of discrete-time nonlinear systems. ISA Trans. 2019, 93, 209-217. [CrossRef]

41. Yang, H.; Xiang, C.; Hao, L.; Zhao, L.; Xue, B. Research on PSA-MFAC for a novel bionic elbow joint system actuated by pneumatic artificial muscles. J. Mech. Sci. Technol. 2017, 31, 3519-3529. [CrossRef]

42. Espinoza-Fraire, T.; Saenz, A.; Salas, F.; Juarez, R.; Giernacki, W. Trajectory Tracking with Adaptive Robust Control for Quadrotor. Appl. Sci. 2021, 11, 8571. [CrossRef]

43. Zhu, X.; Wang, J.; Fang, X.; Zhu, X. Data-driven model-free adaptive sliding mode control for the multi degree-of-freedom robotic exoskeleton. Inf. Sci. 2016, 327, 246-257.

44. Bechlioulis, C.P.; Rovithakis, G.A. Robust adaptive control of feedback linearizable MIMO nonlinear systems with prescribed performance. IEEE Trans. Autom. Control 2008, 53, 2090-2099. [CrossRef] 
45. Liu, D.; Yang, G. Data-Driven Adaptive Sliding Mode Control of Nonlinear Discrete-Time Systems With Prescribed Performance. IEEE Trans. Syst. Man Cybern. Syst. 2019, 49, 2598-2604. [CrossRef]

46. Levant, A. Sliding order and sliding accuracy in sliding mode control. Int. J. Control 1993, 58, 1247-1263. [CrossRef]

47. Teramae, T.; Noda, T.; Hyon, S.H.; Morimoto, J. Modeling and control of a Pneumatic-Electric hybrid system. In Proceedings of the IEEE/RSJ International Conference on Intelligent Robots \& Systems, Tokyo, Japan, 3-8 November 2013; pp. 4887-4892. 